\title{
Laparoscopic fixation of biological mesh at hiatus with glue and suture during hiatal hernia repair
}

\author{
Yusheng Nie, Yao Xiong, Lei Guan, Xin Yuan, Fuqiang Chen, Jie Chen and Huiqi Yang*
}

\begin{abstract}
Background: Laparoscopic paraesophageal hernia repair is associated with higher recurrence rate. Mesh is used to reduce the recurrence rate. This retrospective study is to review our experience of biological mesh fixed with suture and medical glue in hiatal hernias repairs.

Methods: A retrospective chart review was conducted for a consecutive series of patients undergoing laparoscopic hiatal herniorrhaphy between January 2018 and January 2019. After hiatus closure, a piece of biological prosthesis was fixed with medical glue and suture for reinforcement of the crural closure. Clinical outcomes were reviewed, and data were collected regarding operative details, complications, symptoms, and follow-up imaging. Radiological evidence of any size of hiatal hernia was considered to indicate a recurrence.
\end{abstract}

Results: Thirty-six patients underwent surgery uneventfully without any serious complication. There was no mortality. The follow-up was, on average, 18.4 months, and there was no symptomatic recurrence. There was one anatomical recurrence without any related presentation. The method of mesh fixation with medical glue and suture took $12 \mathrm{~min}$ on average, and the handling was fairly easy.

Conclusions: Biological mesh fixed with suture and medical glue was safe and effective for repairing large hiatal hernias. Of course, a longer follow-up is still needed for determining long-term outcomes.

Keywords: Hiatal hernia, Biological mesh, Medical glue

\section{Background}

Hiatal hernia is a common condition often associated with symptomatic gastroesophageal reflux disease (GERD) [1]. Laparoscopic hiatal hernia repair (LHHR) is now considered to be the gold standard for the management of hiatal hernias, and is associated with a reduced rate of perioperative morbidity and shorter hospital stay compared with the open approach [1, 2]. The standard steps include the excision of the sac, a thorough

\footnotetext{
*Correspondence: yang.huiqi@qq.com
}

Department of Hernia and Abdominal Wall Surgery, The Capital Medical University Beijing Chaoyang Hospital, Jingyuan Road No.5, Shijingshan District, Beijing 100043, China oesophageal mobilisation, primary closure of the hiatus, and a fundoplication $[3,4]$.

Oelschlager et al. reported that the recurrence rate after pure suture repair without mesh reinforcement is as high as $59 \%$ at 5 -year follow-up [4]. Primary repair of the paraesophageal hiatal hernia is a significant risk factor for recurrence, especially when suturing the pillars of the diaphragm together under tension for the giant hiatal hernia. Two randomised trials have demonstrated that a significant reduction in recurrence rates can be achieved by using synthetic mesh for large hiatal hernia repair $[5,6]$. However, a few synthetic mesh-related complications, such as mesh erosion, stricture and dysphagia, are reported $[7,8]$. In order to avoid those complications, 
the surgical technique for reliable mesh fixation has been improved, and the mesh specially designed for hiatal hernia has also been improved.

On the other hand, recent studies have reported favourable results from using biological mesh for hiatal hernia repair $[9,10]$. Due to the concern about potential complications related to synthetic mesh, biomaterial was adopted in the repair. However, the long-term results, especially for recurrence, still needs to be investigated further.

In our study, a retrospective study was conducted into laparoscopic hiatal hernia repair at our centre to review our experience with biological mesh for crural reinforcement and on mesh fixation with medical glue and suture.

\section{Methods}

A retrospective study was conducted for a consecutive series of patients undergoing laparoscopic hiatal herniorrhaphy for symptomatic hiatal hernia between January 2018 and January 2019. Preoperative evaluation routinely included endoscopy, CT scan, upper gastrointestinal (UGI) series and oesophageal manometry test, and 24-h $\mathrm{PH}$ monitoring.

\section{Surgical technique}

One dose of antibiotics was administered at induction. Five laparoscopic ports were routinely used. Circumferential dissection of the hernia sac from the hiatus and mediastinal structures was performed. The sac was then first everted over the gastroesophageal junction and then excised. The hiatus was closed posteriorly with an interrupted suture (2-0 Prolene) to about $2.5 \mathrm{~cm}$. A piece of biological prosthesis (ThomalGEN surgical patch, 6*8 cm, Guanhao Biotech Co. Ltd., Guangzhou, China) was prepared and cut in a U configuration, and was fixed with medical glue (Compont medical adhesive, $1.5 \mathrm{ml} /$ tube; Beijing Compont Medical Devices Co. Ltd., Beijing, China) for reinforcement of the crural closure (Fig. 1). The medical glue set consists of a sprayer, catheter, and n-butyl-2-cyanoacrylate (NBCA) glue. The catheter is introduced through one of the operating trocars and a $5 \mathrm{~mm}$ grasper is inserted through the second trocar and used to direct the application of the glue. The mesh is fixed with four-five sprays. In addition, a three-point suture with three intermittent sutures (3-0 vicryl) was used to further strengthen the prosthesis fixation (Fig. 2). Fundoplication was then performed with an interrupted suture (2-0 prolene) [11].

\section{Outcome measurement}

Operative data were collected, including operation time (skin cut to closure), the time cost for prosthesis fixation, blood loss, any intraoperative complication, hiatus

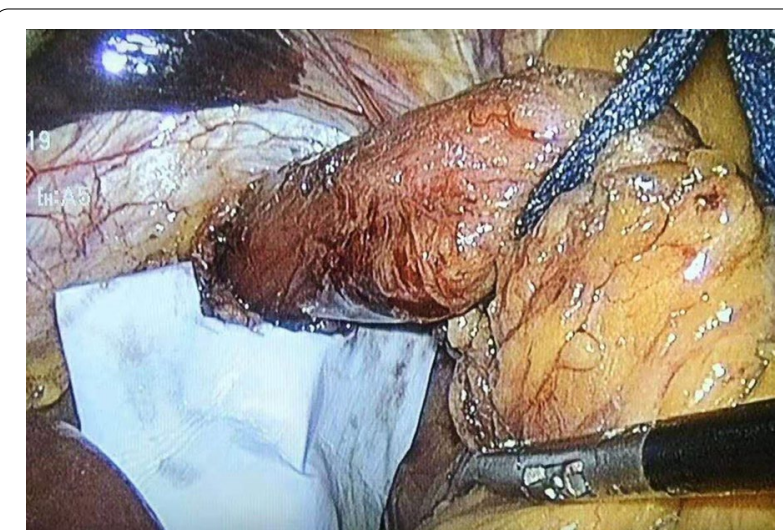

Fig. 1 Initial placement of bioabsorbable mesh with medical glue (Compont Medical Adhesive) applied after cruroplasty

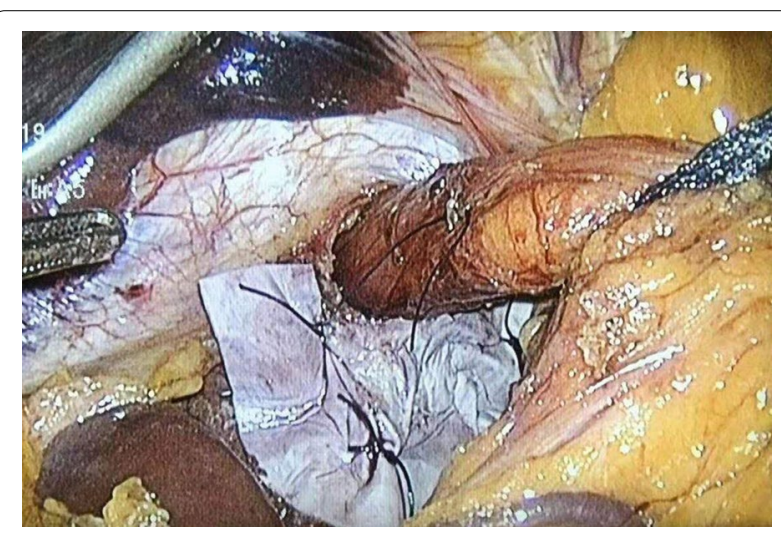

Fig. 2 Three intermittent sutures further fix the prosthesis

measurement, and the length of the intra-abdominal oesophagus after dissection.

Upper Gastrointestinal (UGI) Series: the preoperative and every 6-months postprocedural UGI series were performed at our medical centre. Preoperative exams were interpreted by the clinicians and were used as the primary means of hiatal hernia diagnosis. The radiologists were asked to formulate a consensus interpretation based on the following five-point scale: (1) Intact fundoplication located below the diaphragm without any portions of stomach seen above the plication; (2) Intact fundoplication, but with indeterminate positioning. Plication seen within $2 \mathrm{~cm}$ of the level of the left hemidiaphragm; (3) Intact plication with probable small sliding hiatal hernia. Plication seen between $2 \mathrm{~cm}$ and $5 \mathrm{~cm}$ above the left hemidiaphragm; (4) Intact plication with a large sliding hiatal hernia $(5 \mathrm{~cm}$ above the left hemidiaphragm); (5) Slipped or disrupted fundoplication, and a portion of stomach present above the plication [9]. 


\section{Symptom Questionnaire}

A standardised symptom questionnaire was provided to patients two to four weeks after the operation, 6 months after the operation, and 1 year after the operation. Patients individually scored symptoms based on severity. The symptoms included in the questionnaire were: heartburn, chest pain, dysphagia, and gas bloating. The severity was scored using a visual analogue score (VAS), and patients marked each symptom from 0 to 10 (0 representing no effect on life and 10 representing extreme effect). In addition, the overall satisfaction with the outcome after surgery was scored from 0 to 10 (0 representing not satisfied and 10 representing highly satisfied) as well [11].

Endoscopy was routinely undertaken six months after the operation to evaluate if there was any recurrence.

SPSS 25.0 software was used for statistical analysis.

The protocol for this study was approved by the Clinical Research Ethics Committees at our hospital.

\section{Results}

From January 2018 to January 2019, 36 patients underwent laparoscopic hiatal hernia repair with biological prosthesis fixed by medical glue and suture. Baseline Characteristics of patients is summarised in Table 1 . There were 12 males (33.3\%) and 24 females (66.7\%) with a mean age of $68.4 \pm 17.2$ (range 31-84 years). The average BMI was $28.6 \pm 6.8$. No case underwent previous anti-reflux procedure.

Preoperative oesophageal manometry showed that LESP on average was $6.8 \pm 3.6 \mathrm{mmHg}$, and 12 cases were found to have mild motility disorder. Endoscopy identified the hiatus hernia in all patients, and 7 cases had concomitant esophagitis.

The final diagnosis was confirmed during the operation. Two cases $(5.6 \%)$ were sliding hiatal hernia (type I)with severe acid reflux, five cases (13.9\%) were giant hiatal hernia (type IV) containing at least two-thirds of

Table 1 Baseline characteristics of patients

\begin{tabular}{ll}
\hline & Patients $(\mathrm{n}=36)$ \\
\hline Age $(\mathrm{yr})$ & $68.4 \pm 17.2$ \\
Female & $24(66.7 \%)$ \\
$\mathrm{BMI}\left(\mathrm{kg} / \mathrm{m}^{2}\right)$ & $28.6 \pm 6.8$ \\
Hypertension & $15(41.6 \%)$ \\
Diabetes & $4(11.1 \%)$ \\
Smoking status & $9(25 \%)$ \\
Chronic constipation & $12(33.3 \%)$ \\
COPD & $3(8.3 \%)$ \\
Hospital stay & $4.2 \pm 1.2$ \\
\hline
\end{tabular}

the stomach, and 29 cases (80.5\%) were paraesophageal hernia(type II and type III).

Anethesia evaluation included 31 cases of ASA grade 2 and 5 cases of ASA grade 3 . All the patients underwent laparoscopic surgery uneventfully with an average operation time of $92.6 \mathrm{~min}$ (range 73-135 min). All surgery was performed by one experienced surgeon. The average blood loss was minimal (range $5-20 \mathrm{ml}$ ). The width of hiatus on average was $4.4 \pm 1.4 \mathrm{~cm}$, and the length was $5.2 \pm 1.2 \mathrm{~cm}$. The length of intraabdominal esophagus was more than $3 \mathrm{~cm}$. Fundoplication was performed in all cases. Intraoperative complications were described in four patients (11.1\%): three cases of pneumothorax (8.3\%) during dissection of the sac that could be closed laparoscopically without placement of a chest tube, and one case of superficial liver laceration coagulated laparoscopically.

The mesh placement and fixation took approximately $12 \mathrm{~min}$. The medical glue can provide a very solid fixation to the crural immediately, as the glue can be sprayed quite evenly in small particles. The suture fixation was completed by three intermittent sutures. The prosthesis was well secured, and there was no movement during the following fundoplication.

The analgesia was given when necessary. The patients started to mobilize $6 \mathrm{~h}$ after surgery.

Clinical follow-up is summarised in Tables 2 and 3. The follow-up time was on average 18.4 months, ranging from 13 to 24 months. Overall, the clinical outcome

Table 2 Preoperative and postoperative symptoms assessed using $0-10$ visual analogue scale

\begin{tabular}{llll}
\hline Symptom & Preoperative & 2-4 weeks & $6 \mathrm{~ms}$ \\
\hline Heartburn & $5.20(3.92-6.47)$ & $0.56(0.13-1.00)^{*}$ & $0.48(0.10-1.10)^{*}$ \\
Chest pain & $2.74(1.58-3.88)$ & $0.16(0.02-0.38)^{*}$ & $0.28(0.06-0.44)^{*}$ \\
Regurgitation & $3.62(1.84-4.32)$ & $0.14(0.06-0.20)^{*}$ & $0.18(0.08-0.32)^{*}$ \\
Acid reflux & $5.70(3.82-7.80)$ & $0.12(0.02-0.22)^{*}$ & $0.10(0.02-0.26)^{*}$ \\
Dysphagia & $/$ & $0.57(0.04-1.09)$ & $0.14(0.04-0.22)$ \\
Satisfactory score & $/$ & $8.36(7.20-9.60)$ & $8.18(7.08-9.24)$ \\
\hline
\end{tabular}

All data are expressed as mean $\left(95 \% \mathrm{Cl}\right.$ 's); ${ }^{*} \mathrm{P}<0.01$ Compared to preoperative data

Table 3 Postoperative symptoms assessed using yes versus no questions

\begin{tabular}{lll}
\hline Symptom & $2-4$ weeks (\%) & $6 \mathrm{~ms}$ \\
\hline Heartburn & 0 & $2.78 \%$ \\
Dysphagia & 55.56 & 0 \\
Bloating & 77.78 & $5.56 \%$ \\
Diarrhoea & 13.89 & 0 \\
\hline
\end{tabular}

All data is \% patients 
was favourable. In general, most clinical symptoms such as heartburn,acid reflux, regurgitation, and chest pain improved significantly after the operation. The overall satisfaction score was 8 at 6 months follow up. Even though more than $50 \%$ experienced mild dysphagia and gas bloating after the operation, they improved gradually with diet instruction and all can eat normal diets at 6 months follow-up. No patient needed further intervention, such as endoscopic dilatation or reoperation. Endoscopy at 6 months follow-up, no recurrence was found. Among the 7 cases with concomitant esophagitis, 6 cases was cured, and one case was improved from Grade C to Grade A. At 1-year follow-up, one patient had asymptomatic recurrence shown at Upper GI series, and was scaled as 3 by a radiologist according to the abovementioned criteria (Figs. 3 and 4).

There was no serious postoperative complication, and all were classified into Clavien- Dindo Grade 1. Only one case of asymptomatic recurrence did not need any treatment. No case needed further PPI treatment.

\section{Discussion}

The application of bioabsorbable prosthesis such as Surgisis (small intestinal submucosa, SIS), BioA Tissue Reinforcement (polglycolide or teimethylene carbonate) or AlloDerm (human acellular dermis) in hiatal hernia repair has been suggested to avoid mesh related complications, such as erosion or stricture $[9,12,13]$. In our study, we used a prosthesis made of bovine pericardium for the reinforcement, and this mesh has been widely used in inguinal and ventral hernia repair in China. However, the concern on using biological prosthesis is the weaker strength compared to synthetic material, and this might result in higher recurrence. A few recent studies have confirmed reliable outcomes with biological prosthesis for hiatal hernia repair [13-15]. In our study, including more than $90 \%$ of giant or paraesophageal hernia, there was no symptomatic recurrence during followup. The case of sliding hernia shown at the Upper GI series 1 year after operation did not need further medical intervention. This patient was an 83-year-old female with a giant hiatal hernia, and we were advised to perform the surgery for a shorter time with lower $\mathrm{CO} 2$ pressure by the anaesthetist during the procedure for the safety. Therefore, a simple crural closure was performed without fundoplication, and this could be the reason why the fundus slipped upwards.

In this study, we used NCBA medical glue for prosthesis fixation after crural closure, and the results were satisfactory. The manipulation was easy, and fixation was secure. Previous animal studies have confirmed the safety of chemical and biological adhesives for mesh fixation [16-19]. Moreover, clinical studies have shown that
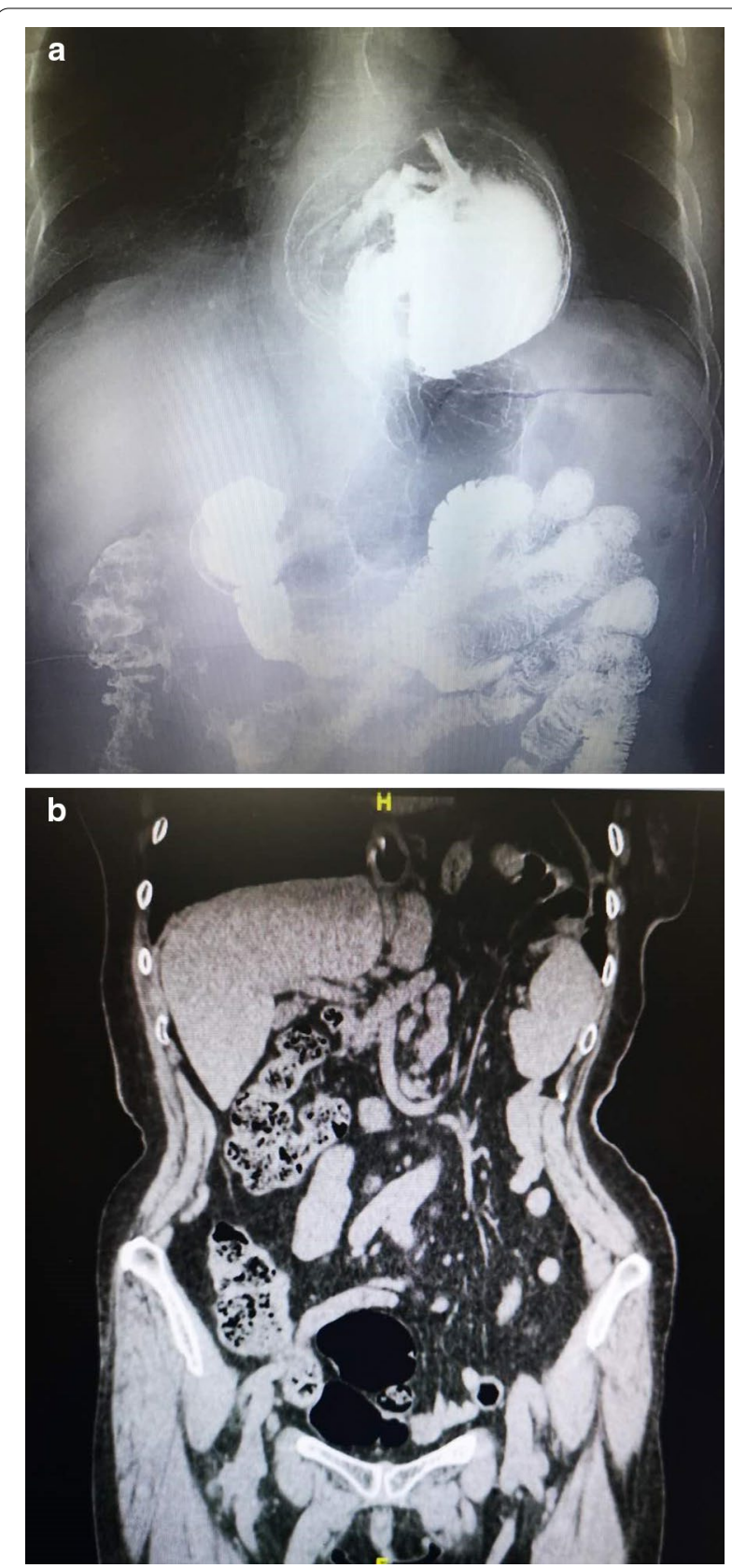

Fig. 3 a, b Preoperative Upper Gl study and CT scan showing a giant hiatal hernia

adhesives, both biological and chemical, are an effective means of mesh fixation in hernia repair and results are comparable to those of traditional techniques such as suture and tack devices [20-27]. However, the shortcomings of biological sealants (e.g., fibrin glue), which are expensive, provide a weak bond, are slow to apply, and are potentially allergenic, have limited their application, particularly in China [7]. For these reasons, Compont, an 

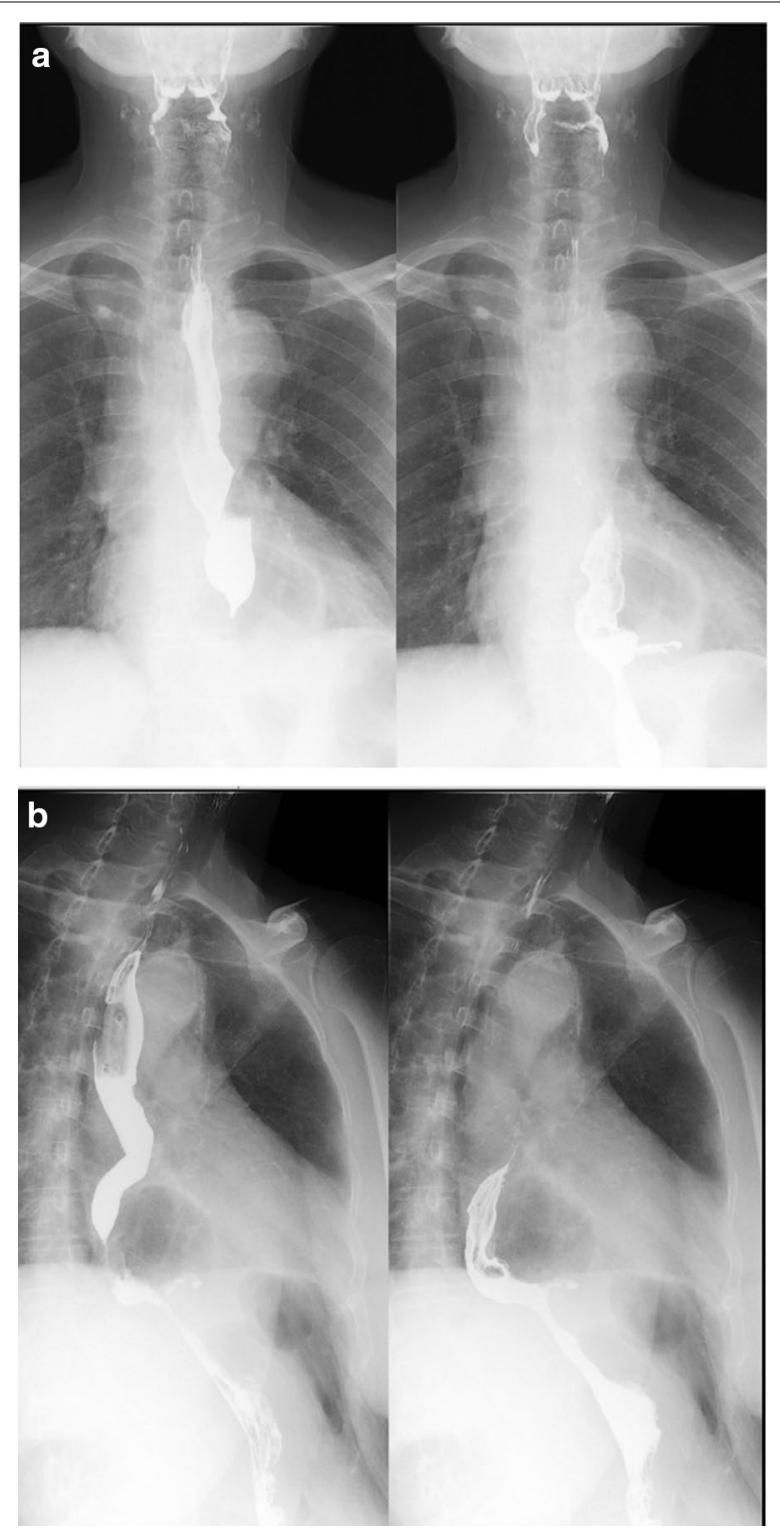

Fig. 4 a, b Upper Gl study at 1 year after the surgery showing sliding hiatal hernia

NBCA chemical adhesive, which is fast acting and provides good adhesive strength, is the preferred surgical adhesive used in China today [28]. The reason why additional suture fixation is needed is that the oesophageal hiatus is a very dynamic area with approximately 3,000 movements every day, therefore erosion, stricture or recurrence might happen with inadequate fixation [12]. However, without glue spray, the fixation needs more sutures, and therefore becomes more time consuming.

Proper mesh fixation is the key for the efficiency and the safety of hiatal hernia repair. Most surgeons currently use suture and some use tacks or staples for fixation. Both of these methods have their problems and do not allow strong, uniform, and immediate fixation of the mesh to the crural fibres. Having used the NCBA glue for mesh fixation in laparoscopic inguinal hernia repair since 2009 at our centre, we noticed that the fixation of the mesh was strong, immediate, and uniform [27]. On the other hand, the application of tacks at hiatus has been reported to cause serious complications such as cardiac tamponade and mortality [29]. As a result, tacker fixation has been strongly advised against by several surgeons [30]. In addition, the SAGES guidelines for management of hiatal hernia state that care should be taken about the mesh fixation technique. In particular, tacks can breach the aorta or pericardium when applied low on the left crus or anteriorly near the apex of the crura [30]. In comparison, medical glue fixation is safe without the risk of penetrating important organs. Furthermore, some studies reported that fixation by glue combined with suture is as strong as tacker [28].

In conclusion, biological mesh reinforcement of crural closure was safe and effective for repairing large hiatal hernias. Medical glue combined with suture can provide solid and secure fixation and can reduce the serious complications caused by fixation. The limitation of this study includes the small number of patients and relatively short follow up period. Ideally, a multiple center clinical study with control group could be carried out for further research.

\section{Conclusions}

Biological mesh fixed with suture and medical glue was safe and effective for repairing large hiatal hernias. Of course, a longer follow-up is still needed for determining long-term outcomes.

\section{Abbreviations}

GERD: Gastroesophageal reflux disease; LHHR: Laparoscopic hiatal hernia repair; UGI: Upper gastrointestinal; NBCA: n-Butyl-2-cyanoacrylate; VAS: Visual analogue score; LESP: Lower esophageal sphincter pressure; SIS: Small intestinal submucosa; Gl: Gastrointestinal; SAGES: Society of American Gsatrointestinal and Endoscopic Surgeons.

\section{Acknowledgements}

Not applicable.

\section{Author contributions}

YN wrote the manuscript. YX, LG, XY and FC collected the data for this study. $\mathrm{HY}$ and JC designed and supervised this study. All authors read and approved the final manuscript.

\section{Funding}

This study was financially supported by the Capital Foundation of Medical Development (2020-2-2035). The funding bodies played no role in the design of the study and collection, analysis, and interpretation of data and in writing the manuscript. 


\section{Availability of data and materials}

The datasets used and/or analysed during the current study are available from the corresponding author on reasonable request.

\section{Declarations}

\section{Ethics approval and consent to participate}

This retrospective chart review study involving human participants was in accordance with the ethical standards of the institutional and national research committee and with the 1964 Helsinki Declaration and its later amendments or comparable ethical standards. The Human Investigation Committee (IRB) of Beijing Chaoyang Hospital approved this study. Written consent was obtained from all the patients to participate this study.

\section{Consent for publication}

Not applicable.

\section{Competing interests}

Dr Nie Yusheng, Yang Huiqi, Xiong Yao, Guan Lei,Yuan Xin,Chen Fuqiang,Chen Jie declare that they have no conflict of interest.

Received: 12 October 2020 Accepted: 11 March 2021

Published online: 22 March 2021

\section{References}

1. Müller-Stich BP, Holzinger F, Kapp T, Klaiber C. Laparoscopic hiatal hernia repair. Surg Endosc Other Interv Tech. 2006;20:380-4.

2. Kohn GP, Price RR, DeMeester SR, et al. Guidelines for the management of hiatal hernia. Surg Endosc. 2013;27:4409-28.

3. Oelschlager BK, Pellegrini CA. Paraesophageal hernias: open, laparoscopic, or thoracic repair? Chest Surg Clin Noth Am. 2001;11:589-603.

4. Oelschlager BK, Pellegrini CA, Hunter J, et al. Biologic prosthesis reduces recurrence after laparoscopic paraesophageal hernia repair: a multicenter, prospective, randomized trial. Ann Surg. 2006;244:481-90.

5. Granderath FA, Schweiger UM, Kamolz T, et al. Laparoscopic Nissen fundoplication with prosthetic hiatal closure reduces postoperative intrathoracic wrap herniation: preliminary results of a prospective randomized functional and clinical study. Arch Surg. 2005;140:40-8.

6. Antoniou SA, Antoniou GA, Koch OO, Pointner R, Granderath FA. Lower recurrence rates after mesh-reinforced versus simple hiatal hernia repair: a meta-analysis of randomized trials. Surg Laparosc Endosc Percutan Tech. 2012;22:498-502.

7. Stadlhuber RJ, Sherif AE, Mittal SK, Fitzgibbons RJ Jr, Brunt M, Hunter L, et al. Mesh complications after prosthetic reinforcement of hiatal closure: a 28-case series. Surg Endosc. 2009;23:1219-26.

8. Gordon AC, Gillespie C, Son J, et al. Long-term outcomes of laparoscopic large hiatus hernia repair with nonabsorbable mesh. Dis Esophagus. 2018:31(5):156.

9. Oelschlager BK, Pellegrini CA, Hunter J, et al. Biologic prosthesis reduces recurrence after laparoscopic paraesophageal hernia repair: a multicenter, prospective, randomized trial. Ann Surg. 2006;244(4):481.

10. Chang CG, Thackeray L. Laparoscopic hiatal hernia repair in 221 patients: outcomes and experience. JSLS. 2016;20(1):e2015.

11. Yang H, Liu Y, Chen J. Clinical efficacy of laparoscopic hiatal herniorrhaphy: a report of 51 cases. Chin J General Surg. 2018;27(10):1273-8.

12. Powell BS, Wandrey D, Voeller GR. A technique for placement of a bioabsorbable prosthesis with fibrin glue fixation for reinforcement of the crural closure during hiatal hernia repair. Hernia. 2013;17(1):81-4.
13. Watkins JR, Truitt MS, Osman H, Jeyarajah RD. Biologic keyhole mesh in hiatal hernia repair. JSLS. 2018;22(1).

14. Sasse KC, Warner DL, Ackerman E, Brandt J. Hiatal hernia repair with novel biological graft reinforcement. JSLS. 2016. https://doi.org/10.4293/JSLS. 2016.00016

15. Antonakis F, Köckerling F, Kallinowski F. Functional results after repair of large hiatal hernia by use of a biologic mesh. Front Surg. 2016;3:16.

16. Jenkins ED, Melman L, Frisella MM, Deeken CR, Matthews BD. Evaluation of acute fifixation strength of absorbable and nonabsorbable barrier coated mesh secured with fibrin sealant. Hernia. 2010;14(5):505-9.

17. Clarke T, Katkhouda N, Mason RJ, Cheng BC, Algra J, Olasky J, et al. Fibrin glue for intraperitoneal laparoscopic mesh fifixation: a comparative study in a swine model. Surg Endosc. 2011;25(3):737-48.

18. Eriksen JR, Bech JI, Linnemann D, Rosenberg J. Laparoscopic intraperitoneal mesh fixation with fibrin sealant $\left(\right.$ Tisseel $^{\circledR}$ ) vs. titanium tacks: a randomised controlled experimental study in pigs. Hernia. 2008;12(5):483-91.

19. Dilege E, Deveci U, Erbil Y, Dinc, c, agA, Seven R, Ozarmagan S, et al. $\mathrm{N}$-butyl cyanoacrylate versus conventional suturing for fifixation of meshes in an incisional hernia model. J Invest Surg. 2010;23(5):262-6.

20. Fortelny RH, Petter-Puchner AH, Glaser KS, Redl H. Use of fibrin sealant (Tisseel/Tissucol) in hernia repair: a systematic review. Surg Endosc. 2012;26(7):1803-12

21. Losi P, Burchielli S, Spiller D, Finotti V, Kull S, Briganti E, et al. Cyanoacrylate surgical glue as an alternative to suture threads for mesh fifixation in hernia repair. J Surg Res. 2010;163(2):e53-8.

22. Fortelny RH, Petter-Puchner AH, May C, Jaksch W, Benesch T, Khakpour $Z$, et al. The impact of atraumatic fibrin sealant vs. staple mesh fixation in TAPP hernia repair on chronic pain and quality of life: results of a randomized controlled study. Surg Endosc. 2012;26(1):249-54.

23. Bittner R, Gmahle E, Gmahle B, Schwarz J, Aasvang E, Kehlet H. Lightweight mesh and noninvasive fixation: an effective concept for prevention of chronic pain with laparoscopic hernia repair (TAPP). Surg Endosc. 2010;24(12):2958-64.

24. Schafer M, Vuilleumier H, Di Mare L, Demartines N. Fibrin sealant for mesh fifixation in endoscopic inguinal hernia repair: is there enough evidence for its routine use? Surg Laparosc Endosc Percutan Tech. 2010;20(4):205-12.

25. Testini M, Lissidini G, Poli E, Gurrado A, Lardo D, Piccinni G. A singlesurgeon randomized trial comparing sutures, Nbutyl-2-cyanoacrylate and human fibrin glue for mesh fifixation during primary inguinal hernia repair. Can J Surg. 2010;53(3):155-60.

26. Zonta S, Lovisetto F, Longoni M. Use of human fibrin glue (tissucol) versus staples for mesh fixation in laparoscopic transabdominal preperitoneal hernioplasty. Ann Surg. 2007;246(5):903-4.

27. Wang M, Tian M, Zhao X, Nie Y, Chen J, Shen Y. Effectiveness and safety of n-butyl-2-cyanoacrylate medical adhesive for noninvasive patch fixation in laparoscopic inguinal hernia repair. Surg Endosc. 2013;27:3792-8.

28. Jenkins ED, Melman L, Desai S, Brown SR, Frisella MM, Deeken CR, Matthews BD. Evaluation of intraperitoneal placement of absorbable and nonabsorbable barrier coated mesh secured with fibrin sealant in a New Zealand white rabbit model. Surg Endosc. 2011;25:604-12.

29. Frantzides CT, Welle SN. Cardiac tamponade as a life-threatening complication in hernia repair. Surgery. 2012;152:133-5.

30. Köckerling F, Schug-Pass C, Bittner R. A word of caution: never use tacks for mesh fixation to the diaphragm. Surg Endosc. 2018;32:3295-302.

\section{Publisher's note}

Springer Nature remains neutral with regard to jurisdictional claims in published maps and institutional affiliations. 